\title{
COINCIDENCE AND FIXED POINT THEOREMS IN METRIC AND BANACH SPACES
}

\author{
ZEQING LIU, JEONG SHEOK UME, and MOHAMMAD S. KHAN
}

(Received 7 August 2000)

\begin{abstract}
We prove some coincidence and fixed point theorems for generalized $(\phi, f)$ contractive type and $f$-nonexpansive type multivalued and singlevalued mappings in metric and Banach spaces, respectively. Our results extend, improve, and unify a few known results due to Browder, Browder and Petryshyn, Chang, Huang, and Cho, Husain and Latif, Husain and Tarafdar, and Karlovitz.
\end{abstract}

2000 Mathematics Subject Classification. 54H25, 47H10.

1. Introduction. Husain and Latif $[5,6,7]$ introduced the notions of $f$-contractive type and $f$-nonexpansive type multivalued mappings and proved fixed point theorems for the mappings in metric and Banach spaces, respectively. Chang, Huang, and Cho [4] introduced the concept of generalized $f$-contractive type multivalued mappings in metric spaces and established a coincidence point theorem for generalized $f$-contractive type multivalued mappings.

Motivated and inspired by the above works, we introduce a concept of generalized $(\phi, f)$-contractive type multivalued mappings which contains the concepts of $f$ contractive type multivalued mappings and generalized $f$-contractive type multivalued mappings as special cases, and we prove three coincidence and fixed point theorems for such mappings which extend and improve the main results of Chang, Huang, and Cho [4], Husain and Latif [6, 7], and others. On the other hand, we study the existence of common fixed points of $f$-nonexpansive type multivalued mappings which generalizes the main results of Husain and Latif $[5,6,7]$ and contains some results due to Browder [1], Browder and Petryshyn [2], Husain and Tarafdar [8], and Karlovitz [9] as particular cases.

2. Preliminaries. Throughout this paper, we denote by $\mathbb{R}, \mathbb{R}^{+}, \mathbb{N}$ the set of real numbers, the set of nonnegative real numbers, and the set of positive integers, respectively. Let $\Phi$ be the family of mappings $\phi:\left(\mathbb{R}^{+}\right)^{5} \rightarrow \mathbb{R}^{+}$such that each $\phi$ is nondecreasing for each variable and

$$
\psi(t) \geq \max \{\phi(t, t, t, a t, b t): a, b \in\{0,1,2\}\}, \quad \forall t \in \mathbb{R}^{+},
$$

where $\psi: \mathbb{R}^{+} \rightarrow \mathbb{R}^{+}$is nondecreasing, right continuous, $\sum_{n=1}^{\infty} \psi^{n}(t)<\infty$ for all $t \in \mathbb{R}^{+}$ and $\lim _{n \rightarrow \infty}\left(t_{n}-\psi\left(t_{n}\right)\right)=0$ implies that $\lim _{n \rightarrow \infty} t_{n}=0$ for any $\left\{t_{n}\right\}_{n \in \mathbb{N}} \subset \mathbb{R}^{+}$.

LEMmA 2.1 (see [3]). Let $h: \mathbb{R}^{+} \rightarrow \mathbb{R}^{+}$be nondecreasing, right continuous, and $\lim _{n \rightarrow \infty} h^{n}(t)=0$ for all $t \in \mathbb{R}^{+}$. Then 
(i) $h(t)<t$ for all $t>0$;

(ii) $t=0$ provided that $t \leq h(t)$ for some $t \in \mathbb{R}^{+}$.

LEMMA 2.2. Let $\left\{a_{n}\right\}_{n \in \mathbb{N}}$ and $\left\{b_{n}\right\}_{n \in \mathbb{N}}$ be in $\mathbb{R}^{+}$and $\lim _{n \rightarrow \infty} a_{n}=0$. Then

$$
\limsup _{n \rightarrow \infty}\left(a_{n}+b_{n}\right)=\limsup _{n \rightarrow \infty} b_{n} \text {. }
$$

Proof. Note that

$$
\limsup _{n \rightarrow \infty} b_{n} \leq \limsup _{n \rightarrow \infty}\left(a_{n}+b_{n}\right) \leq \limsup _{n \rightarrow \infty} a_{n}+\limsup _{n \rightarrow \infty} b_{n}=\limsup _{n \rightarrow \infty} b_{n},
$$

which implies that (2.2) holds. This completes the proof.

LEMMA 2.3. Let $\left\{a_{n}\right\}_{n \in \mathbb{N}}$ be a sequence in $\mathbb{R}^{+}$and $h: \mathbb{R}^{+} \rightarrow \mathbb{R}^{+}$be nondecreasing and right continuous. Then

$$
\limsup _{n \rightarrow \infty} h\left(a_{n}\right) \leq h\left(\limsup _{n \rightarrow \infty} a_{n}\right) .
$$

Proof. Set $\limsup _{n \rightarrow \infty} h\left(a_{n}\right)=a$. It follows that there exists a subsequence $\left\{a_{n_{i}}\right\}_{i \in \mathbb{N}}$ of $\left\{a_{n}\right\}_{n \in \mathbb{N}}$ with $\lim _{i \rightarrow \infty} h\left(a_{n_{i}}\right)=a$. Put $\limsup _{i \rightarrow \infty} a_{n_{i}}=b$. Then there exists a subsequence $\left\{a_{n_{i_{j}}}\right\}_{j \in \mathbb{N}}$ of $\left\{a_{n_{i}}\right\}_{i \in \mathbb{N}}$ such that $\lim _{j \rightarrow \infty} a_{n_{i_{j}}}=b$. Observe that each sequence in $\mathbb{R}$ contains a monotone subsequence. Thus $\left\{a_{n_{i_{j}}}\right\}_{j \in \mathbb{N}}$ contains a monotone subsequence $\left\{a_{n_{i_{j k}}}\right\}_{k \in \mathbb{N}}$. If $\left\{a_{n_{i_{j k}}}\right\}_{k \in \mathbb{N}}$ is nondecreasing, then $h\left(a_{n_{i_{j k}}}\right) \leq h(b)$ for any $k \in \mathbb{N}$. This means that

$$
\lim _{k \rightarrow \infty} h\left(a_{n_{i j k}}\right) \leq h(b) .
$$

If $\left\{a_{n_{i j k}}\right\}_{k \in \mathbb{N}}$ is nonincreasing, by the right continuity of $h$, we obtain that

$$
\lim _{k \rightarrow \infty} h\left(a_{n_{i_{j k}}}\right)=h\left(\lim _{k \rightarrow \infty} a_{n_{i_{j k}}}\right)=h(b) .
$$

Using (2.5) and (2.6), we have

$$
a \leq h(b) \leq h\left(\limsup _{n \rightarrow \infty} a_{n}\right) .
$$

That is, (2.4) holds. This completes the proof.

Let $(X, d)$ be a metric space and $M$ a nonempty subset of $X$. Define

$$
\begin{aligned}
2^{M} & =\{A: A \text { is nonempty subset of } M\}, \\
\mathrm{CL}(M) & =\{A: A \text { is nonempty closed subset of } M\}, \\
\mathrm{CB}(M) & =\{A: A \text { is nonempty closed bounded subset of } M\}, \\
C(M) & =\{A: A \text { is nonempty compact subset of } M\} .
\end{aligned}
$$

Let $H$ denote the Hausdorff metric on $\mathrm{CB}(X)$ induced by $d$. That is,

$$
H(A, B)=\max \left\{\sup _{x \in A} d(x, B), \sup _{x \in B} d(x, A)\right\}, \quad \forall A, B \in \mathrm{CB}(X),
$$

where $d(x, A)=\inf \{d(x, y): y \in A\}$. 
DeFinITION 2.4. Let $r$ be in [0,1), $\phi \in \Phi$, and let $J: M \rightarrow 2^{X}$ and $f: M \rightarrow X$ be multivalued and singlevalued mappings, respectively.

(i) $J$ is said to be a generalized $(\phi, f)$-contractive type multivalued mapping if, for all $x \in M$ and for each $u_{x} \in J x$, there exists $v_{y} \in J y$ for all $y \in M$ satisfying

$$
d\left(u_{x}, v_{y}\right) \leq \phi\left(d(f x, f y), d\left(f x, u_{x}\right), d\left(f y, v_{y}\right), d\left(f x, v_{y}\right), d\left(f y, u_{x}\right)\right) .
$$

(ii) $J$ is said to be a generalized $f$-contractive type multivalued mapping if, for all $x \in M$ and for each $u_{x} \in J x$, there exists $v_{y} \in J y$ for all $y \in M$ satisfying

$$
d\left(u_{x}, v_{y}\right) \leq r \max \left(d(f x, f y), d\left(f x, u_{x}\right), d\left(f y, v_{y}\right), \frac{1}{2}\left[d\left(f x, v_{y}\right)+d\left(f y, u_{x}\right)\right]\right)
$$

(iii) $J$ is said to be an $f$-contractive type multivalued mapping if, for all $x \in M$ and for each $u_{x} \in J x$, there exists $v_{y} \in J y$ for all $y \in M$ satisfying

$$
d\left(u_{x}, v_{y}\right) \leq r d(f x, f y)
$$

(iv) $J$ is said to be an $f$-nonexpansive type multivalued mapping if, for all $x \in M$ and for each $u_{x} \in J x$, there exists $v_{y} \in J y$ for all $y \in M$ satisfying

$$
d\left(u_{x}, v_{y}\right) \leq d(f x, f y)
$$

Clearly, each $f$-contractive type multivalued mapping is both $f$-nonexpansive type multivalued mapping and generalized $f$-contractive type multivalued mapping, and each generalized $f$-contractive type multivalued mapping is a generalized $(\phi, f)$ contractive type multivalued mapping.

3. Coincidence and fixed points of generalized $(\phi, f)$-contractive type multivalued mappings. In this section, we establish three coincidence and fixed point theorems for generalized $(\phi, f)$-contractive type multivalued mappings in complete metric space.

THEOREM 3.1. Let $M$ be a nonempty closed subset of a complete metric space $(X, d)$, and let $f: M \rightarrow M$ be a singlevalued mapping, and $J: M \rightarrow \mathrm{CL}(M)$ a generalized $(\phi, f)$ contractive type multivalued mapping. Suppose that $f M=M$. Then $f$ and $J$ have a coincidence point in $M$.

Proof. Let $x_{0}$ be an arbitrary but fixed element of $M$ and choose $y_{1} \in J x_{0}$. It follows from $M=f M$ that there exists $x_{1} \in M$ with $y_{1}=f x_{1}$. Since $J$ is a generalized $(\phi, f)$-contractive type multivalued mapping and $M=f M$, we can choose $y_{2} \in J x_{1}$ and $x_{2} \in M$ such that

$$
d\left(y_{1}, y_{2}\right) \leq \phi\left(d\left(f x_{0}, f x_{1}\right), d\left(f x_{0}, y_{1}\right), d\left(f x_{1}, y_{2}\right), d\left(f x_{0}, y_{2}\right), d\left(f x_{1}, y_{1}\right)\right)
$$

and $y_{2}=f x_{2}$. Inductively, we can extract a sequence $\left\{f x_{n}\right\}_{n \in \mathbb{N}} \subset M$ such that $f x_{n+1}$ 
is in $J x_{n}$ and

$$
\begin{aligned}
d_{n} & \leq \phi\left(d_{n-1}, d_{n-1}, d_{n}, d\left(f x_{n-1}, f x_{n+1}\right), d\left(f x_{n}, f x_{n}\right)\right) \\
& \leq \phi\left(d_{n-1}, d_{n-1}, d_{n}, d_{n-1}+d_{n}, 0\right),
\end{aligned}
$$

where $d_{n}=d\left(f x_{n}, f x_{n+1}\right)$ for all $n \geq 0$. We claim that

$$
d_{n} \leq d_{n-1}, \quad \forall n \in \mathbb{N} .
$$

Suppose that $d_{n}>d_{n-1}$ for some $n \in \mathbb{N}$. Thus (3.2) ensures that $d_{n} \leq \psi\left(d_{n}\right)$. It follows from Lemma 2.1 that $d_{n}=0$, which is a contradiction. Therefore (3.3) holds. In view of (3.2) and (3.3), we have

$$
d_{n} \leq \psi\left(d_{n-1}\right) \leq \cdots \leq \psi^{n}\left(d_{0}\right), \quad \forall n \in \mathbb{N}
$$

Thus for all $n, p \in \mathbb{N}$, we conclude that

$$
d\left(f x_{n}, f x_{n+p}\right) \leq \sum_{i=n}^{n+p-1} d_{i} \leq \sum_{i=n}^{n+p-1} \psi^{i}\left(d_{0}\right) .
$$

Note that $\sum_{n=1}^{\infty} \psi^{n}\left(d_{0}\right)<\infty$. It follows from (3.5) that $\left\{f x_{n}\right\}_{n \in \mathbb{N}}$ is a Cauchy sequence in the closed set $M$. Since $X$ is complete, there exists $z \in M$ such that $\lim _{n \rightarrow \infty} f x_{n}=z$. Observe that $f M=M$. Hence there exists $q \in M$ with $f q=z$. From $f x_{n+1} \in J x_{n}$, $f M=M$, and (2.10), we can choose that $f v_{n} \in J q$ satisfying

$$
\begin{aligned}
& d\left(f x_{n+1}, f v_{n}\right) \\
& \quad \leq \phi\left(d\left(f x_{n}, f q\right), d\left(f x_{n}, f x_{n+1}\right), d\left(f q, f v_{n}\right), d\left(f x_{n}, f v_{n}\right), d\left(f q, f x_{n+1}\right)\right) \\
& \leq \psi\left(\max \left\{d\left(f x_{n}, f q\right), d\left(f x_{n}, f x_{n+1}\right), d\left(f q, f v_{n}\right), d\left(f x_{n}, f v_{n}\right), d\left(f q, f x_{n+1}\right)\right\}\right) .
\end{aligned}
$$

It is easy to see that there exists an increasing sequence $\left\{n_{i}\right\}_{i \in \mathbb{N}} \subset \mathbb{N}$ such that at least one of (3.7), (3.8), and (3.9) holds,

$$
\begin{aligned}
d\left(f x_{n_{i}+1}, f v_{n_{i}}\right) \leq & \psi\left(\max \left\{d\left(f x_{n_{i}}, f q\right), d\left(f x_{n_{i}}, f x_{n_{i}+1}\right), d\left(f q, f v_{n_{i}}\right)\right\}\right), \quad \forall i \in \mathbb{N} \\
& d\left(f x_{n_{i}+1}, f v_{n_{i}}\right) \leq \psi\left(d\left(f x_{n_{i}}, f v_{n_{i}}\right)\right), \quad \forall i \in \mathbb{N} \\
& d\left(f x_{n_{i}+1}, f v_{n_{i}}\right) \leq \psi\left(d\left(f q, f x_{n_{i}+1}\right)\right), \quad \forall i \in \mathbb{N} .
\end{aligned}
$$

We now prove that

$$
\lim _{i \rightarrow \infty} d\left(f q, f v_{n_{i}}\right)=0 .
$$

Suppose that (3.7) is satisfied. If $\lim _{i \rightarrow \infty} d\left(f q, f v_{n_{i}}\right) \neq 0$, then there exist $\varepsilon>0$ and a subsequence $\left\{v_{n_{i_{j}}}\right\}_{J \in \mathbb{N}}$ of $\left\{v_{n_{i}}\right\}_{i \in \mathbb{N}}$ such that $d\left(f q, f v_{n_{i j}}\right) \geq \varepsilon$ for all $j \in \mathbb{N}$. Note that 
$\lim _{n \rightarrow \infty} f x_{n}=f q$ and $\lim _{n \rightarrow \infty} d\left(f x_{n}, f x_{n+1}\right)=0$. Thus there exists $k \in \mathbb{N}$ satisfying $\max \left\{d\left(f x_{n_{i_{j}}}, f q\right),\left(f x_{n_{i_{j}}}, f x_{n_{i_{j}}+1}\right)\right\}<\varepsilon$ for all $j \geq k$. Using (3.7), we have

$$
\begin{aligned}
d\left(f q, f v_{n_{i_{j}}}\right) \leq & d\left(f q, f x_{n_{i_{j}}+1}\right)+d\left(f x_{n_{i_{j}}+1}, f v_{n_{i_{j}}}\right) \\
\leq & d\left(f q, f x_{n_{i_{j}}+1}\right) \\
& +\psi\left(\max \left\{d\left(f x_{n_{i_{j}}}, f q\right), d\left(f x_{n_{i_{j}}}, f x_{n_{i_{j}}+1}\right), d\left(f q, f v_{n_{i_{j}}}\right)\right\}\right) \\
\leq & d\left(f q, f x_{n_{i_{j}}+1}\right)+\psi\left(\max \left\{\varepsilon, \varepsilon, d\left(f q, f v_{n_{i_{j}}}\right)\right\}\right) \\
\leq & d\left(f q, f x_{n_{i_{j}}+1}\right)+\psi\left(d\left(f q, f v_{n_{i_{j}}}\right)\right),
\end{aligned}
$$

for all $j \geq k$. Equation (3.11) ensures that

$$
d\left(f q, f v_{n_{i_{j}}}\right)-\psi\left(d\left(f q, f v_{n_{i_{j}}}\right)\right) \leq d\left(f q, f x_{n_{i_{j}}+1}\right) \longrightarrow 0 \text { as } j \rightarrow \infty,
$$

which implies that $\lim _{j \rightarrow \infty} d\left(f q, f \nu_{n_{i_{j}}}\right)=0$. This is a contradiction. Consequently, (3.10) holds.

Suppose that (3.8) is fulfilled. In view of (3.8), we obtain that for all $i \in \mathbb{N}$,

$$
\begin{aligned}
d\left(f q, f v_{n_{i}}\right) & \leq d\left(f q, f x_{n_{i}+1}\right)+d\left(f x_{n_{i}+1}, f v_{n_{i}}\right) \\
& \leq d\left(f q, f x_{n_{i}+1}\right)+\psi\left(d\left(f x_{n_{i}}, f v_{n_{i}}\right)\right) \\
& \leq d\left(f q, f x_{n_{i}+1}\right)+\psi\left(d\left(f x_{n_{i}}, f q\right)+d\left(f q, f v_{n_{i}}\right)\right) .
\end{aligned}
$$

By virtue of (3.13) and Lemmas 2.2 and 2.3, we conclude that

$$
\begin{aligned}
\limsup _{i \rightarrow \infty} d\left(f q, f v_{n_{i}}\right) & \leq \limsup _{i \rightarrow \infty}\left[d\left(f q, f x_{n_{i}+1}\right)+\psi\left(d\left(f x_{n_{i}}, f q\right)+d\left(f q, f v_{n_{i}}\right)\right)\right] \\
& =\limsup _{i \rightarrow \infty} \psi\left(d\left(f x_{n_{i}}, f q\right)+d\left(f q, f v_{n_{i}}\right)\right) \\
& \leq \psi\left(\limsup _{i \rightarrow \infty}\left[d\left(f x_{n_{i}}, f q\right)+d\left(f q, f v_{n_{i}}\right)\right]\right) \\
& =\psi\left(\limsup _{i \rightarrow \infty} d\left(f q, f v_{n_{i}}\right)\right) .
\end{aligned}
$$

Using (3.14) and Lemma 2.1, we get that $\limsup _{i \rightarrow \infty} d\left(f q, f v_{n_{i}}\right)=0$. It is easy to see that (3.10) holds.

Suppose that (3.9) is satisfied. Then

$$
\begin{aligned}
d\left(f q, f v_{n_{i}}\right) & \leq d\left(f q, f x_{n_{i}+1}\right)+d\left(f x_{n_{i}+1}, f v_{n_{i}}\right) \\
& \leq d\left(f q, f x_{n_{i}+1}\right)+\psi\left(d\left(f q, f x_{n_{i}+1}\right)\right) \rightarrow 0 \text { as } i \rightarrow \infty .
\end{aligned}
$$

That is, (3.10) holds. 
Since $J q$ is closed and $\left\{f v_{n_{i}}\right\}_{i \in \mathbb{N}} \subset J q$, by (3.10) we conclude immediately that $f q$ belongs to $J q$. This completes the proof.

Taking $\phi(u, v, w, x, y)=r \max \{u, v, w,(1 / 2)(x+y)\}$ in Theorem 3.1, we have the following corollary.

COROLlary 3.2 (see [4, Theorem 2.1]). Let $M$ be a nonempty closed subset of a complete metric space $(X, d), f: M \rightarrow M$ a singlevalued mapping, and $J: M \rightarrow \operatorname{CL}(M)$ a generalized $f$-contractive type multivalued mapping. Suppose that $f M=M$. Then $f$ and $J$ have a coincidence point in $M$.

REMARK 3.3. Theorem 2.2 of [4] and Theorem 2.3 of [6] are special cases of Theorem 3.1.

To see that the closedness of $M$ in the hypothesis of Theorem 3.1 is essential, we need the following example.

EXAMPLE 3.4. Let $X=\mathbb{R}$ with the usual metric. Take $M=(0,1), J x=\left\{(1 / 4)\left(1-x^{4}\right)\right\}$, and $f x=1-x^{2}$ for all $x \in M$. Obviously, $|J x-J y| \leq(1 / 2)|f x-f y|$ for all $x, y \in M$. The requirements of Theorem 3.1 are satisfied except that $M$ is closed. But $f$ and $J$ have no coincidence point in $M$.

The following example reveals that the condition of $f M=M$ in Theorem 3.1 is necessary.

EXAMPLE 3.5. Let $X=\mathbb{R}$ with the usual metric and $M=[0,1]$. Define $J x=\left\{(1 / 6) x^{2}\right\}$ and $f x=1-(1 / 2) x$ for all $x, y \in M$. Then $|J x-J y| \leq(2 / 3)|f x-f y|$ for all $x, y \in M$. It is easy to see that the hypotheses of Theorem 3.1 are satisfied except $f M=M$. However, $f$ and $J$ have no coincidence point in $M$.

THEOREM 3.6. Let $M$ be a nonempty closed subset of a complete metric space $(X, d)$, and let $f: M \rightarrow M$ a singlevalued mapping and $J: M \rightarrow \mathrm{CL}(M)$ a generalized $(\phi, f)$ contractive type multivalued mapping. If $f$ is continuous, $J M \subseteq f M$, and $f J x \subseteq J f x$ for all $x \in M$, then $f$ and $J$ have a coincidence point in $M$.

Proof. Let $\left\{f x_{n}\right\}_{n \in \mathbb{N}}$ and $z$ be as in the proof of Theorem 3.1. Put $u_{n}=f x_{n}$ for all $n \in \mathbb{N}$. Since $\lim _{n \rightarrow \infty} f x_{n}=z \in M$ and $f$ is continuous in $M$, so that $f u_{n}=f f x_{n} \rightarrow f z$ as $n \rightarrow \infty$. From $u_{n} \in J x_{n-1}$, we have $f u_{n} \in f J x_{n-1} \subseteq J f x_{n-1}=J u_{n-1}$. It follows from (2.10) that there exists $v_{n} \in J z$ satisfying

$$
\begin{aligned}
& d\left(f u_{n}, v_{n}\right) \\
& \quad \leq \phi\left(d\left(f u_{n-1}, f z\right), d\left(f u_{n-1}, f u_{n}\right), d\left(f z, v_{n}\right), d\left(f u_{n-1}, v_{n}\right), d\left(f z, f u_{n}\right)\right)
\end{aligned}
$$

As in the proof of Theorem 3.1, we conclude that there exists a subsequence $\left\{u_{n_{i}}\right\}_{i \in \mathbb{N}}$ of $\left\{u_{n}\right\}_{n \in \mathbb{N}}$ such that $\lim _{i \rightarrow \infty} u_{n_{i}}=f z$ and $f z \in J z$. This completes the proof.

REMARK 3.7. In case $f=i_{X}$ (the identity mapping on $X$ ), Theorem 3.6 reduces to a result which extends and improves Theorem 2.3 of [6]. 
THEOREM 3.8. Let $X, M, f$, and $J$ be as in Theorem 3.6. Suppose that for all $x \in M$, the following condition is fulfilled:

$$
f x \in J x \text { implies } \lim _{n \rightarrow \infty} f^{n} x \text { exists. }
$$

Then $f$ and $J$ have a common fixed point in $M$.

Proof. Theorem 3.6 ensures that there exists $z \in X$ such that $f z \in J z$. Set $f^{n} z=$ $a_{n}$ for all $n \in \mathbb{N}$. Equation (3.17) and the closedness of $M$ means that there exists $t \in M$ such that $a_{n} \rightarrow t$ as $n \rightarrow \infty$. Since $f$ is continuous, $f a_{n}=f^{n+1} z \rightarrow t=f t$ as $n \rightarrow \infty$. Observe that

$$
a_{n}=f^{n} z=f^{n-1} f z \in f^{n-1} J z \subseteq J f^{n-1} z=J a_{n-1}, \quad \forall n \in \mathbb{N} .
$$

It follows from (2.10) that there exists $b_{n} \in J t$ satisfying

$$
\begin{aligned}
d\left(a_{n}, b_{n}\right) & \leq \phi\left(d\left(f a_{n-1}, f t\right), d\left(f a_{n-1}, a_{n}\right), d\left(f t, b_{n}\right), d\left(f a_{n-1}, b_{n}\right), d\left(f t, a_{n}\right)\right) \\
& \leq \phi\left(d\left(a_{n}, t\right), 0, d\left(t, b_{n}\right), d\left(a_{n}, t\right)+d\left(t, b_{n}\right), d\left(t, a_{n}\right)\right) \\
& \leq \psi\left(d\left(a_{n}, t\right)+d\left(t, b_{n}\right)\right),
\end{aligned}
$$

which implies that

$$
d\left(t, b_{n}\right) \leq d\left(t, a_{n}\right)+d\left(a_{n}, b_{n}\right) \leq d\left(t, a_{n}\right)+\psi\left(d\left(a_{n}, t\right)+d\left(t, b_{n}\right)\right) .
$$

Put $S=\lim \sup _{n \rightarrow \infty} d\left(b_{n}, t\right)$. Using (3.20) and Lemmas 2.2 and 2.3, we obtain that

$$
\begin{aligned}
S & \leq \limsup _{n \rightarrow \infty} d\left(a_{n}, t\right)+\limsup _{n \rightarrow \infty} \psi\left(d\left(a_{n}, t\right)+d\left(b_{n}, t\right)\right) \\
& \leq \psi\left(\limsup _{n \rightarrow \infty} d\left(a_{n}, t\right)+\limsup _{n \rightarrow \infty} d\left(b_{n}, t\right)\right)=\psi(S) .
\end{aligned}
$$

Lemma 2.1 implies that $S=0$. Therefore $b_{n} \rightarrow t$ as $n \rightarrow \infty$. Since $J t$ is closed and $\left\{b_{n}\right\}_{n \in \mathbb{N}} \subset J t$, we conclude that $t$ belongs to $J t$. This completes the proof.

Husain and Latif [5] and Kaneko and Sessa [9] proved the following results, respectively.

THEOREM 3.9 (see [5, Theorem 2.2]). Let $M$ be a nonempty closed subset of a complete metric space $(X, d)$ and $f$ a continuous mapping of $M$ into itself. Suppose $J: M \rightarrow$ $\mathrm{CL}(M)$ is an $f$-contractive type multivalued mapping such that $J f=f J$ and $J M \subseteq f M$. Moreover, assume that one of (3.17) and (3.22) holds for all $x \in M$, where

$$
f x \neq f^{2} x \text { implies } f x \notin J x \text {. }
$$

Then $f$ and $J$ have a common fixed point in $M$.

THEOREM 3.10 (see [9, Theorem 3]). Let $(X, d)$ be a complete metric space, and let $f$ : $X \rightarrow X$ and $J: X \rightarrow \mathrm{CB}(X)$ be compatible continuous mappings such that $J X \subseteq f X$ and

$$
H(J x, J y) \leq r \max \left\{d(f x, f y), d(f x, J x), d(f y, J y), \frac{1}{2}[d(f x, J y)+d(f y, J x)]\right\},
$$

for any $x, y \in X$, where $0 \leq r<1$. Assume also that for each $x \in X$ either (3.17) or (3.22) holds. Then $f$ and $T$ have a common fixed point in $X$. 
REMARK 3.11. Note that (3.22) is equivalent to the following

$$
f x \in J x \text { implies } f x=f^{2} x \text {. }
$$

It is easy to see that (3.22) implies (3.17). Therefore (3.22) in Theorems 3.9 and 3.10 is superfluous. Thus Theorem 2.2 of [5] is a special case of Theorem 3.8.

REMARK 3.12. It follows from [5, Theorem 4.2, Condition (A)] that for any two mappings $J_{i}, J_{j}$ and any $x \in M, J_{i} x \subseteq J_{j} x$, and $J_{j} x \subseteq J_{i} x$. That is, $J_{i} x=J_{j} x$ for all $x \in M$, and $i, j \in \mathbb{N}$. Thus [5, Theorem 4.2] is the same as [5, Theorem 2.2].

4. Fixed points of $f$-nonexpansive type multivalued mappings. In this section, we establish a common fixed point theorem for $f$-nonexpansive type multivalued mappings in nonempty convex weakly compact subsets of a Banach space under certain conditions.

A Banach space $(X,\|\cdot\|)$ is said to satisfy Opial's condition [11] if for each $x \in X$ and each sequence $\left\{x_{n}\right\}_{n \in \mathbb{N}}$ weakly converging to $x$,

$$
\liminf _{n \rightarrow \infty}\left\|x_{n}-y\right\|>\liminf _{n \rightarrow \infty}|| x_{n}-x \|, \quad \forall y \neq x .
$$

THEOREM 4.1. Let $M$ be a nonempty weakly compact convex subset of a Banach space $X$ which satisfies Opial's condition. Suppose that $f$ is a continuous affine mapping of $M$ into itself and $J: M \rightarrow C(M)$ is an $f$-nonexpansive type multivalued mapping satisfying

$$
\begin{gathered}
J M \subseteq f M, \\
f J x \subseteq J f x, \quad \forall x \in M .
\end{gathered}
$$

Then $f$ and $J$ have a common fixed point in $M$.

Proof. We may, without loss of generality, assume that $0 \in M$. For each sequence $\left\{h_{n}\right\}_{n \in \mathbb{N}}$ with $h_{n} \in(0,1)$ and $\lim _{n \rightarrow \infty} h_{n}=1$, we define $J_{n} x=\left\{h_{n} u: u \in J x\right\}$ for $x \in M$. It follows from (4.3) that

$$
J_{n} f x \supseteq\left\{h_{n} u: u \in f J x\right\}=\left\{f t: t \in h_{n} J x\right\}=f J_{n} x .
$$

As in the proof of Theorem 3.1 in [6], we conclude that $J_{n}: M \rightarrow \operatorname{CL}(M)$ is an $f$ contractive type multivalued mapping and $J_{n} M \subseteq f M$ for all $n \in \mathbb{N}$. Let $k \in \mathbb{N}$. We show that if $f x$ is in $J_{k} x$, then $\lim _{n \rightarrow \infty} f^{n} x$ exists. Assume that $f x \in J_{k} x=h_{k} J x$. Then there exists $u_{1} \in J x$ with $f x=h_{k} u_{1}$. This implies that $f u_{1} \in f J x \subseteq J f x=J h_{k} u_{1}=h_{k} J u_{1}$ and $f u_{1}=h_{k} u_{2}$ for some $u_{2} \in J u_{1}$. Consequently, $f^{2} x=f h_{k} u_{1}=h_{k} f u_{1}=h_{k}^{2} u_{2}$. Using (4.3), we can construct a sequence $\left\{u_{n}\right\}_{n \in \mathbb{N}} \subseteq M$ with $u_{n+1} \in J u_{n}$ and $f u_{n}=$ $h_{k} u_{n+1}$ for all $n \geq 0$, where $u_{0}=x$. It is clear that

$$
f^{n} x=f^{n-1} f x=f^{n-1} h_{k} u_{1}=f^{n-2} h_{k}^{2} u_{2}=\cdots=h_{k}^{n} u_{n}, \quad \forall n \in \mathbb{N} .
$$

Since $M$ is bounded and $\left\{u_{n}\right\}_{n \in \mathbb{N}} \subseteq M$, it follows that $\left\{\left\|u_{n}\right\|\right\}_{n \in \mathbb{N}}$ is bounded. Hence $\left\|f^{n} x\right\|=h_{k}^{n}\left\|u_{n}\right\| \rightarrow 0$ as $n \rightarrow \infty$. In view of Theorem 3.8, we obtain that $f$ and $J_{n}$ have a common fixed point in $M$. The remaining part of the proof is as in Theorem 3.1 of [6]. This completes the proof.

As a consequence of Theorem 4.1, we have the following corollary. 
COROllary 4.2 (see [6, Theorem 3.1]). Let $M, X, f, J$ be as in Theorem 4.1. Suppose that $f$ and $J$ satisfy (4.2) and the following relations:

$$
\begin{gathered}
f J x=J f x, \quad \forall x \in M \\
f x \neq f^{2} x \text { implies } t f x \notin J x, \quad \forall x \in M, t \geq 1 .
\end{gathered}
$$

Then $f$ and $J$ have a common fixed point in $M$.

REMARK 4.3. Theorem 4.1 extends Theorems 2.2 and 3.2 of [5] and Theorem 3.4 of [7]. Theorem 4.1 contains also the results due to Browder [1], Browder and Petryshyn [2], and Karlovitz [10], respectively, as particular cases.

ACKNOWLEDGEMENT. The second author was supported by grant No. 2001-110100-005-2 from the Basic Research Program of the Korea Science and Engineering Foundation.

\section{REFERENCES}

[1] F. E. Browder, Fixed-point theorems for noncompact mappings in Hilbert space, Proc. Nat. Acad. Sci. U.S.A. 53 (1965), 1272-1276. MR 31\#2582. Zbl 125.35801.

[2] F. E. Browder and W. V. Petryshyn, Construction of fixed points of nonlinear mappings in Hilbert space, J. Math. Anal. Appl. 20 (1967), 197-228. MR 36\#747. Zbl 153.45701.

[3] S. S. Chang, Fixed point theorems for single-valued and multi-valued mappings in nonArchimedean Menger probabilistic metric spaces, Math. Japon. 35 (1990), no. 5, 875-885. MR 91m:54058. Zbl 711.54033.

[4] S. S. Chang, N. J. Huang, and Y. J. Cho, Coincidence and fixed point theorems, Bull. Honam Math. Soc. 12 (1995), 153-161.

[5] T. Husain and A. Latif, Fixed points of multivalued nonexpansive maps, Math. Japon. 33 (1988), no. 3, 385-391. MR 89i:47107. Zbl 667.47028.

[6] _ _ f f-nonexpansive type maps and fixed points, Math. Japon. 36 (1991), no. 6, 10531061. CMP 1139 723. Zbl 755.54020.

[7] _ Fixed points of multivalued nonexpansive maps, Int. J. Math. Math. Sci. 14 (1991), no. 3, 421-430. MR 92k:54056. Zbl 736.54030.

[8] T. Husain and E. Tarafdar, Fixed point theorems for multivalued mappings of nonexpansive type, Yokohama Math. J. 28 (1980), no. 1-2, 1-6. MR 82m:47039. Zbl 456.47047.

[9] H. Kaneko and S. Sessa, Fixed point theorems for compatible multi-valued and singlevalued mappings, Int. J. Math. Math. Sci. 12 (1989), no. 2, 257-262. MR 90i:54097. Zbl 671.54023.

[10] L. A. Karlovitz, On nonexpansive mappings, Proc. Amer. Math. Soc. 55 (1976), no. 2, 321325. MR 53\#8976. Zbl 328.47033.

[11] E. Lami Dozo, Multivalued nonexpansive mappings and Opial's condition, Proc. Amer. Math. Soc. 38 (1973), 286-292. MR 46\#9816. Zbl 268.47060.

Zeqing LiU: Department of MATHEMATics, LiaOning NoRmal UniVersity, Dalian, LIAONING 116029, CHINA

Jeong Sheok Ume: Department of ApPlied Mathematics, Changwon National UniverSITY, CHANGWON 641-773, KOREA

E-mail address: jsume@sarim.changwon.ac.kr

Mohammad S. Khan: Department of Mathematics and Statistics, College of Science, Sultan QABOOS UNIVERSITY, AL-KHOUd, MusCAT 123, OMAN

E-mail address: mohammad@squ . edu . om 


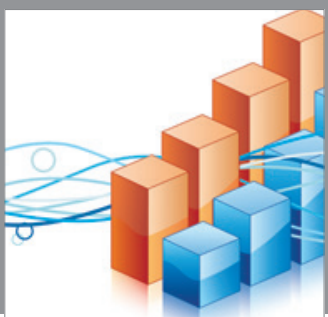

Advances in

Operations Research

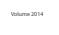

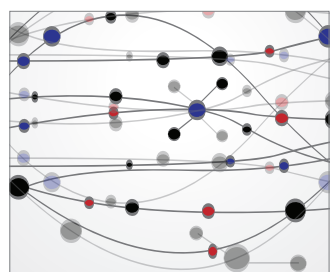

\section{The Scientific} World Journal
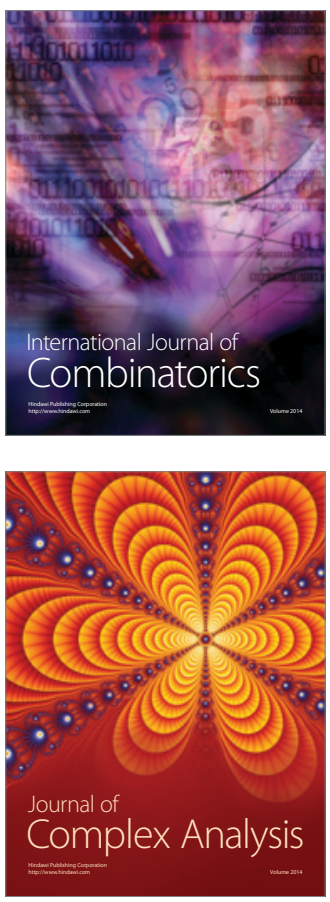

International Journal of

Mathematics and

Mathematical

Sciences
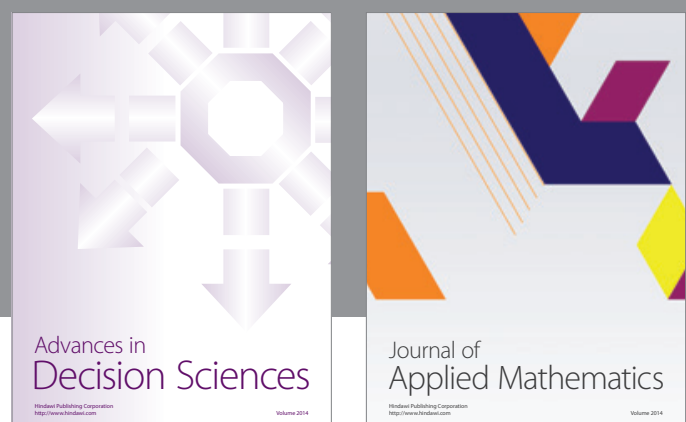

Journal of

Applied Mathematics
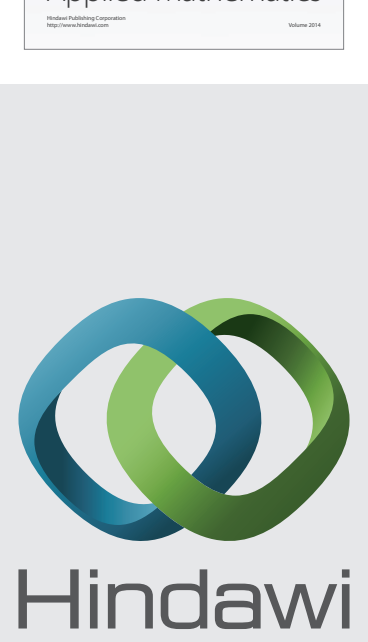

Submit your manuscripts at http://www.hindawi.com
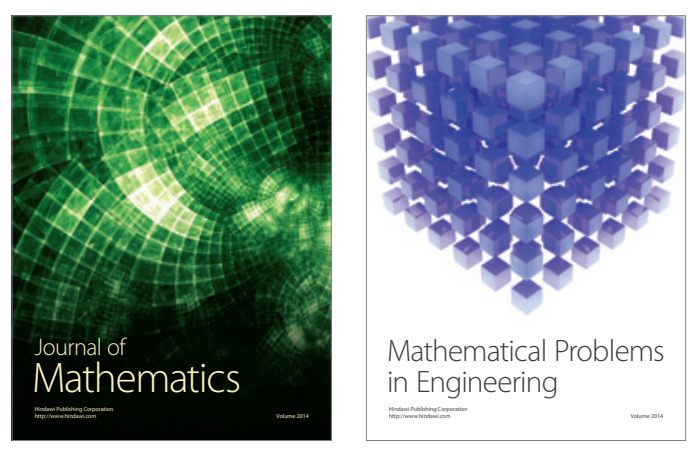

Mathematical Problems in Engineering
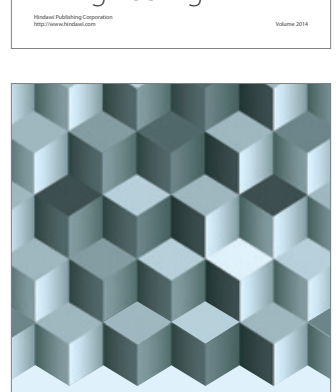

Journal of

Function Spaces
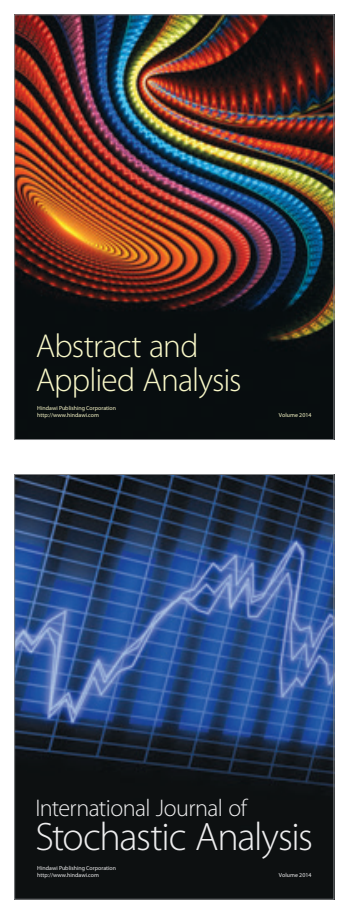

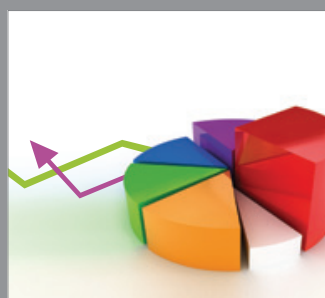

ournal of

Probability and Statistics

Promensencen
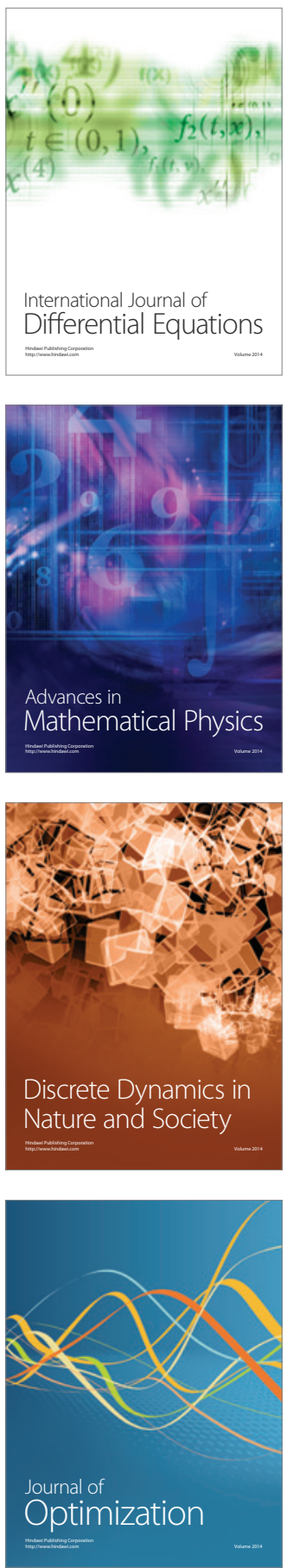